\title{
Multi-wavelength Behavior of Blazars: Combined Order \& Disorder
}

\author{
Alan P. Marscher ${ }^{* \dagger}$ \\ Institute for Astrophysical Research, Boston University, 725 Commonwealth Ave., Boston, MA \\ 02215 USA \\ E-mail: marscher@bu.edu
}

\begin{abstract}
Blazars display behavior that contains both apparently random elements, such as flux variations that follow a red-noise power spectrum, and systematic phenomena like apparent superluminal knots of emission that propagate for some distance down the jet. Models involving turbulent plasma flowing down a relativistic jet, punctuated by standing and moving shocks, show promise in explaining this dichotomy. One such framework, the author's Turbulent, Extreme Multi-Zone (TEMZ) model, produces artificial multi-waveband flux and polarization versus time curves that bear considerable resemblance to data from monitoring studies of blazars. Here the author presents some of these results. Wavelength-dependent optical polarization predicted by the TEMZ model can provide a definitive test of the hypothesis that turbulence is the main cause of rapid fluctuations in blazar emission.
\end{abstract}

4th Annual Conference on High Energy Astrophysics in Southern Africa

25-27 August, 2016

Cape Town, South Africa

\footnotetext{
* Speaker.

${ }^{\dagger}$ The research summarized here was supported by NASA Fermi Guest Investigator grant NNX14AQ58G, NASA Swift Guest Investigator grant NNX15AR45G, and US National Science Foundation grant AST-1615796.
} 


\section{Introduction}

Attempts to understand the nature of blazars tend to lead toward frustration. Analysis of a particular event, such as a flare, can provide a clear description that can be explained by a physically motivated model, while a second such event differs enough that a similar model fails to reproduce its behavior. Still, our observations find some order as well. The emitting plasma is funneled into a very narrow jet down which high-energy plasma flows at near-light speeds away from the central black hole. Bright knots of emission that often have the properties of shock waves appear to propagate down the jet at superluminal velocities. A stationary bright spot is found at the upstream end of the jet in very long baseline interferometry (VLBI) images at millimeter wavelengths [8] with polarization properties similar to those predicted for conical standing "recollimation" shocks $[4,5]$. At optical wavelengths, the linear polarization vector can rotate rather smoothly by as much as $720^{\circ}[13,14,2]$.

A successful theory of blazar emission must be capable of explaining this superposition of order and disorder. The author has been attempting to accomplish this with his Turbulent Extreme Multi-Zone (TEMZ) model [12], in which the magnetic field in the jet changes from nearly 100\% helical to mostly disordered, and then becomes partially re-ordered by one or more shock waves. The following sections contain a summary of the features of this model and how they can potentially explain the observed properties of blazars.
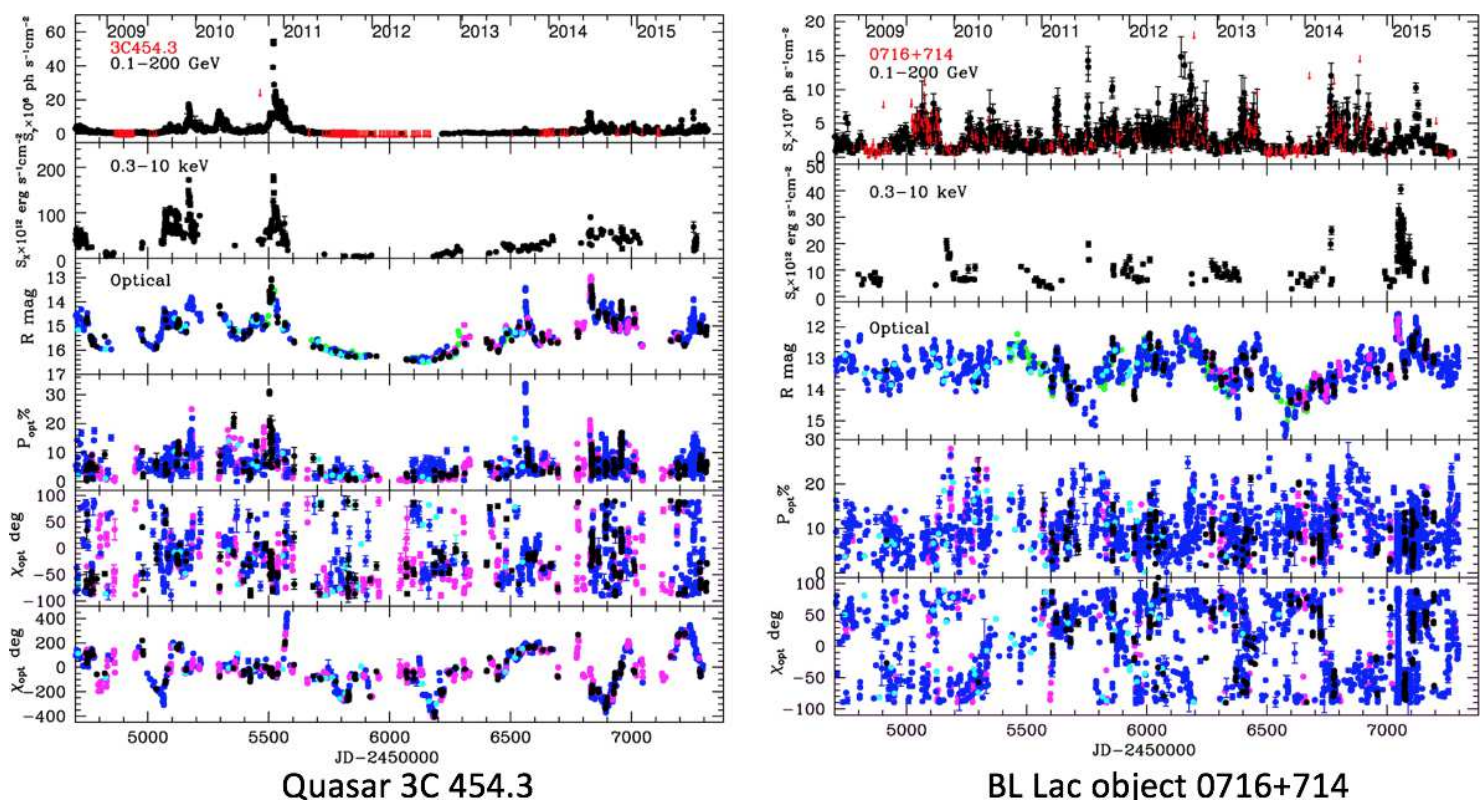

Figure 1: Multi-waveband flux and optical linear polarization vs. time of the quasar 3C 454.3 (left) and the BL Lac object $0716+714$ (right). For 3C 454.3, the bottom panel allows the polarization position angle $\chi$ to extend beyond the -90 to $+90^{\circ}$ range by minimizing the difference between successive values; this accentuates apparent rotations in the polarization vector. From data presented in [22] and papers in preparation) 


\section{Observed Characteristics of Blazars}

Figure 1 presents flux and linear polarization vs. time at multiple wavebands for two quintessential blazars, the quasar 3C 454.3 and the BL Lac object 0716+714. The data are from an international collaboration led by the Boston University blazar research group. Some of these data have been published in [22], where a description of the telescopes and data analysis can be found. More recent data displayed in Figure 1 (as well as Fig. 3 below) will be published in future papers.

One obvious difference between the two blazars is in the $\gamma$-ray light curve. The quasar exhibits intervals of wild outbursts and prolonged periods of relative quiescence, with a flux ratio between the brightest and faintest states greater than 2 orders of magnitude. In contrast, the BL Lac object varies by factor $\sim 15$, without lengthy time spans of non-detections. Both objects, though, have highly variable optical polarization, with an electric-vector position angle $\chi$ that sometimes fluctuates about the jet direction (usually $\sim-80^{\circ}$ in $3 \mathrm{C} 454.3$ during the period under consideration and $\sim 90^{\circ}$ in $0716+714$ ), other times fluctuates wildly, and occasionally rotates rather smoothly. The flux variations have various amplitudes and time-scales as time progresses, and indeed they can usually be characterized as red noise [6].

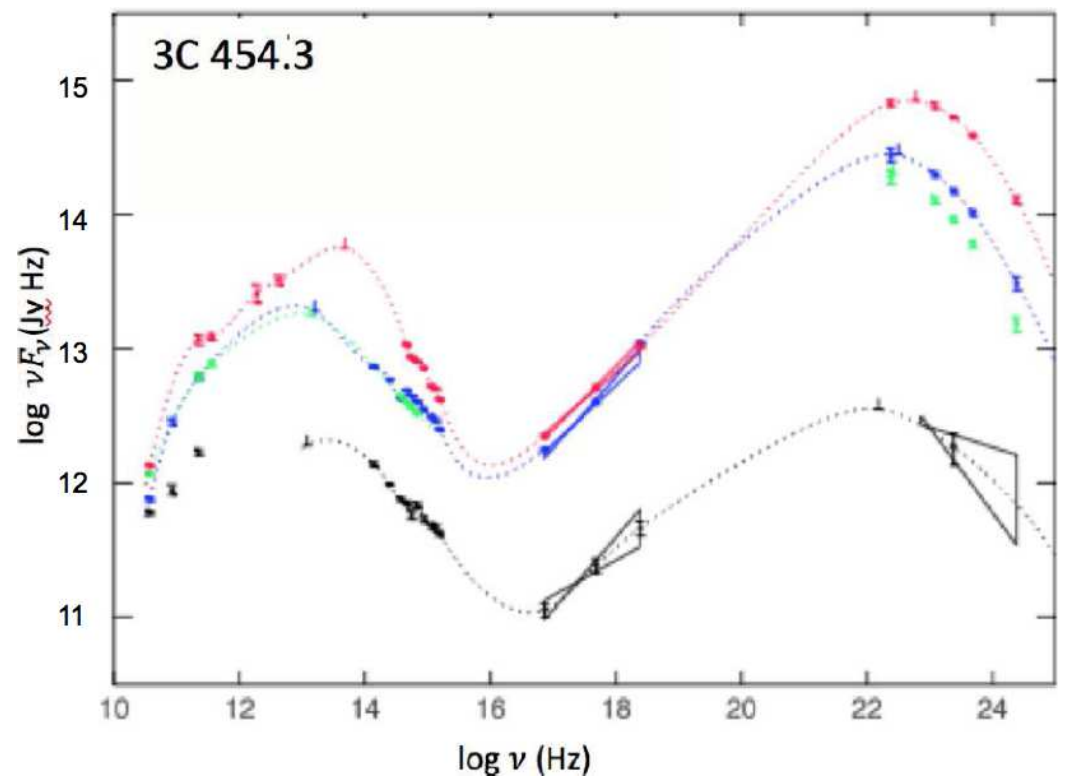

Figure 2: Example of a spectral energy distribution of a blazar: the quasar 3C 454.3, measured at four times — three during outbursts and one during relative quiescence; from [10]

As illustrated in Figure 2, the spectral energy distribution (SED) of a blazar generally has two peaks, one customarily associated with synchrotron radiation (peaking in the IR region in Fig. 2) and the other probably with inverse Compton scattering (peaking at $\gamma$-ray frequencies in Fig. 2). There are at least two breaks in the synchrotron SED: one at millimeter wavelengths as the entire jet apparently becomes optically thin, and the other at IR to UV wavelengths where there is an effective steepening of the slope of the electron energy spectrum. This latter break is usually by more than $1 / 2$, the value expected from radiative losses [15]. 


\section{Application of the TEMZ Model}

The TEMZ model [12] takes a new approach to the simulation of blazar light curves and polarization behavior. The key ingredient is the turbulent component of the magnetic field, which adds randomness to the systematic components of blazar jets - narrowly focused relativistic flow, partially ordered magnetic field, and shocks. The radiating particles are assumed to be present in the ambient jet, accelerated by the second-order Fermi mechanism and by numerous magnetic reconnections in the turbulent regions, after which their energies are boosted further by the shock(s). The amount of the boost depends on the angle between the magnetic field and the shock normal (see, e.g., [19]). This causes the highest energy electrons to be present over limited volumes; this energydependent filling factor steepens the spectrum and enhances the variability at higher frequencies (owing to the smaller emitting volume). The steepening is by more than $1 / 2$, as observed, and the spectrum can deviate from a power law above the break, similar to the log-parabolic spectrum often used in analyses of the $\gamma$-ray spectra of blazars [1].

The TEMZ code varies the energy density in particles at the jet input randomly within a powerlaw power spectrum. Simulated turbulence is superposed on these variations via nested zones as follows. The jet is divided into thousands of cells of plasma, each with a uniform magnetic field and energy distribution of relativistic electrons. Following the scheme of Jones [7], each cell belongs to several nested zones of different dimensions ( 1 cell, $2 \times 2 \times 2$ cells, $4 \times 4 \times 4$ cells, and $8 \times 8 \times 8$ cells). Each cell moves at a randomly directed turbulent velocity (e.g., at the local sound speed) superposed on the systemic velocity of the jet flow. The relative contribution of each zone to a given cell's magnetic field and relativistic electron density follows a Kolmogorov spectrum. This scheme gives each cell a unique turbulent magnetic field component while maintaining a correlation with the field in neighboring cells, as is generally the case in actual turbulence. The energies of the relativistic electrons are boosted by an extra factor when they cross a shock front, with the amount of the boost greater if the magnetic field of the cell is nearly parallel to the shock normal in the plasma frame (the "subluminal" regime of diffusive shock acceleration). The shock also compresses both the component of the magnetic field that is transverse to the shock normal and the density of the particles.

The code computes the synchrotron radiation from each cell, including the polarization, as a function of time and frequency. The calculation includes the dependence of the emission coefficient on the direction of the magnetic field relative to the (relativistically aberrated) line of sight. This is a major factor in the variations of flux and polarization. The inverse Compton flux varies as the number of relativistic electrons and their energies, as well as the density of seed photons, change. The seed photons can be from hot dust in the nuclear region, from synchrotron radiation in the jet (which can arise in relatively slowly moving regions such as a sheath or Mach disk, or in other cells moving with the same systemic velocity plus their own turbulent velocities), or from emission-line clouds.

Figure 3 displays light curves and optical polarization vs. time of BL Lacertae, data which provide a goal for the TEMZ model to replicate in a general manner. Figures 4 and 5 present, respectively, multi-waveband flux and both optical and millimeter-wave polarization vs. time curves produced by sample simulations of the TEMZ code. This is only an illustration, since the input parameters were not optimized to produce the best possible fit to the BL Lacertae data. 


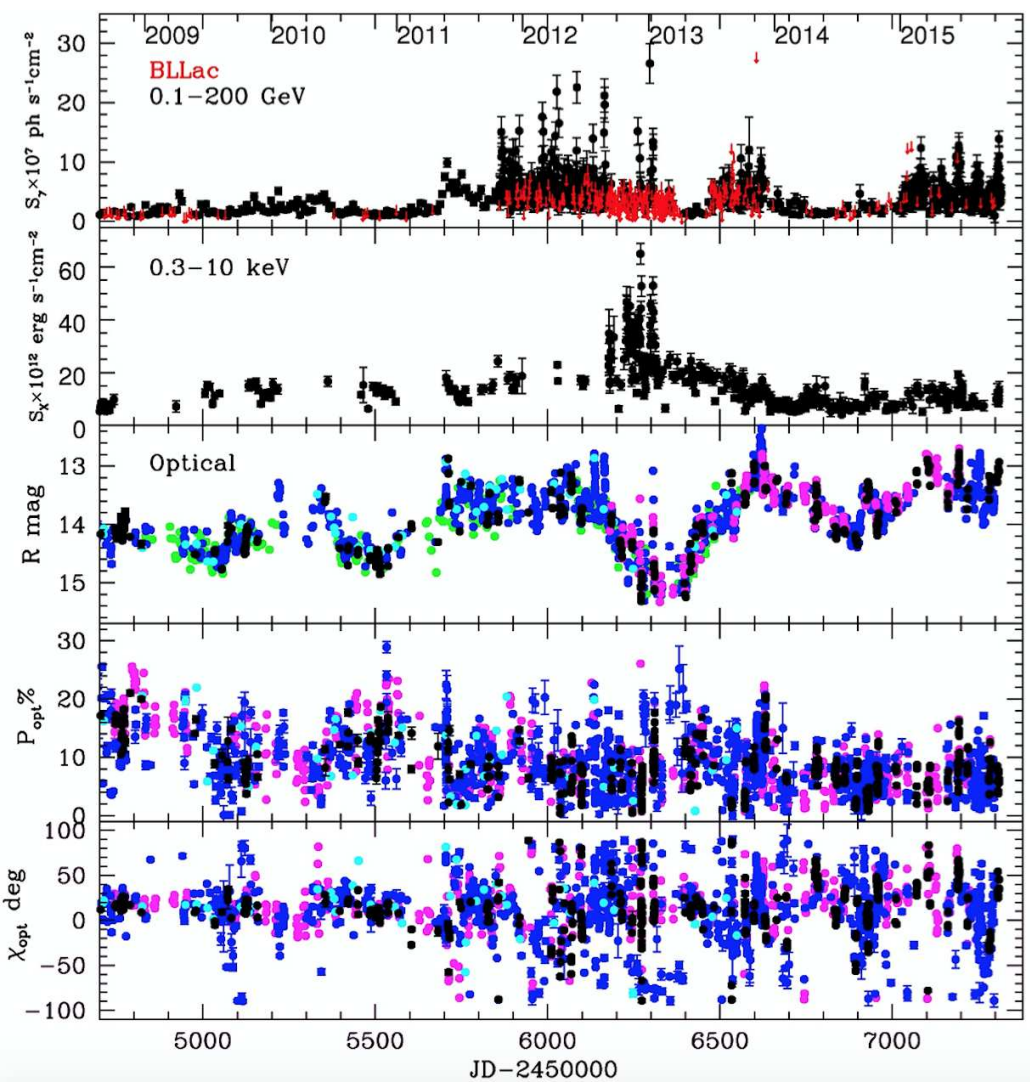

Figure 3: Multi-waveband flux and optical linear polarization vs. time of BL Lacertae. From data to be presented in [21] and papers in preparation)
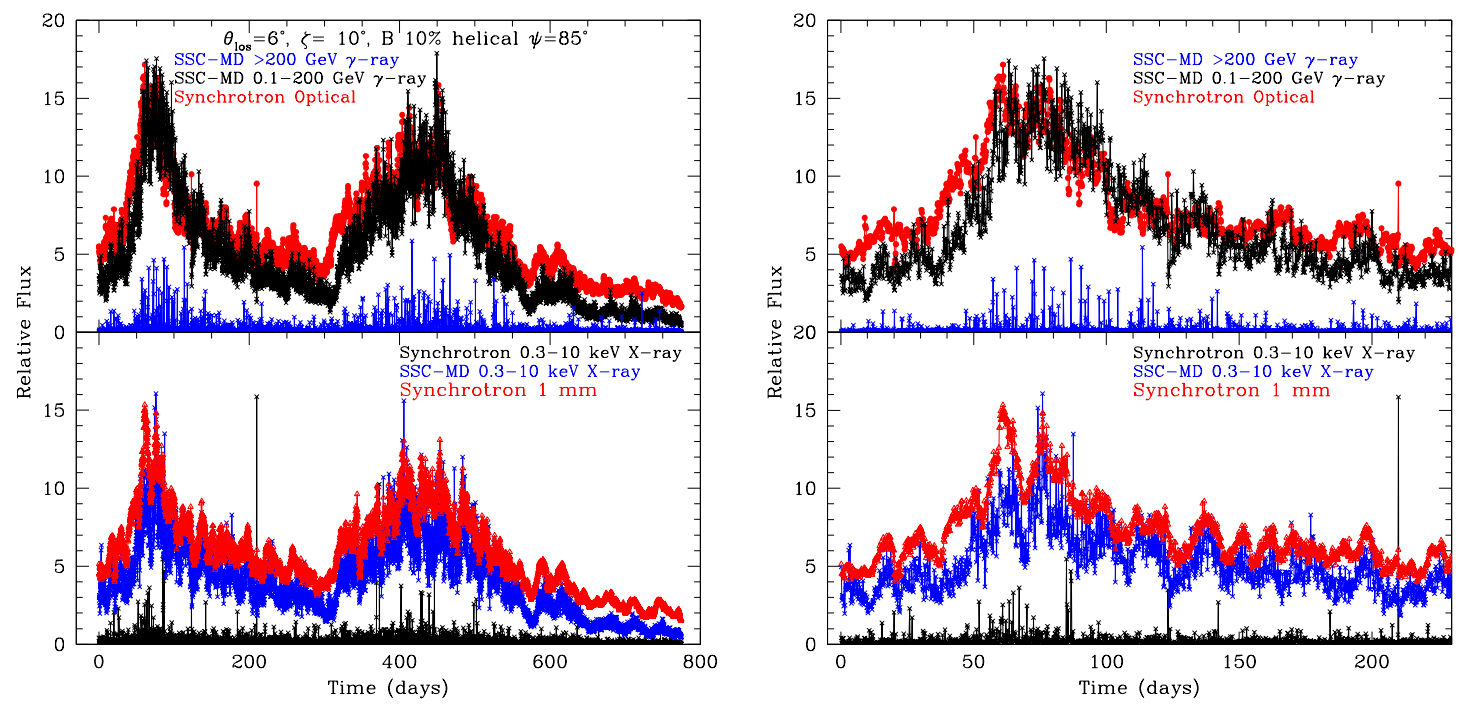

Figure 4: Simulated multi-waveband flux vs. time produced by the TEMZ model for parameters similar to those of BL Lacertae. Left: Full run of 5000 time steps, with each time step equivalent to 0.155 days; right: expansion of the section containing the first flare. Compare with Fig. 3. 
The simulations have a number of features that are similar to observed characteristics of blazars described in the previous section. Note in particular the overall tendency of the flux at different wavebands to vary together as activity in the jet waxes and wanes, yet short time-scale details do not correspond closely. The range of $\gamma$-ray variations is greater than that of the optical variations, a feature that would be accentuated if steadier components of the optical emission were included. (This is especially true for quasars, for which the big blue bump provides a minimum optical flux level.) The variation in degree of polarization and the tendency of the position angle to fluctuate about the jet direction $\left(0^{\circ}\right.$ or $180^{\circ}$ in the simulations and $\sim 190^{\circ}$ in BL Lacertae [8]), with occasional rotations, is also seen in the actual data.

The time resolution of the TEMZ code corresponds to how long in the observer's frame it takes a turbulent cell to cross its own length. In the simulation displayed in Figures 4 and 5, this is 0.155 days for a source at the distance of BL Lacertae. Factor of $\sim 2$ intraday changes of flux and polarization are common, caused by turbulent cells with favorable field directions (both for particle acceleration and near-unity sine of the angle of the field to the aberrated line of sight) and high field values crossing the shock front. (Some very high-amplitude flares over a single time step are seen, which suggests that the log-normal distribution of the turbulent magnetic field and density should probably be truncated at some maximum level.) The peculiar motions of the cells play a role as well: even for sonic turbulent motions (as opposed to the supersonic motions previously proposed to explain rapid variability [16]) at $c / \sqrt{3}$, the Lorentz factor is increased (or decreased) by a factor as high as 1.9 relative to the systemic value (see also [3]). The $>200 \mathrm{GeV}$ $\gamma$-ray emission displayed in Figure 4 is highly sporadic, with detectable levels generally seen only over single time steps, as is the case observationally for quasars and low-synchrotron-peak BL Lac objects such as BL Lacertae.
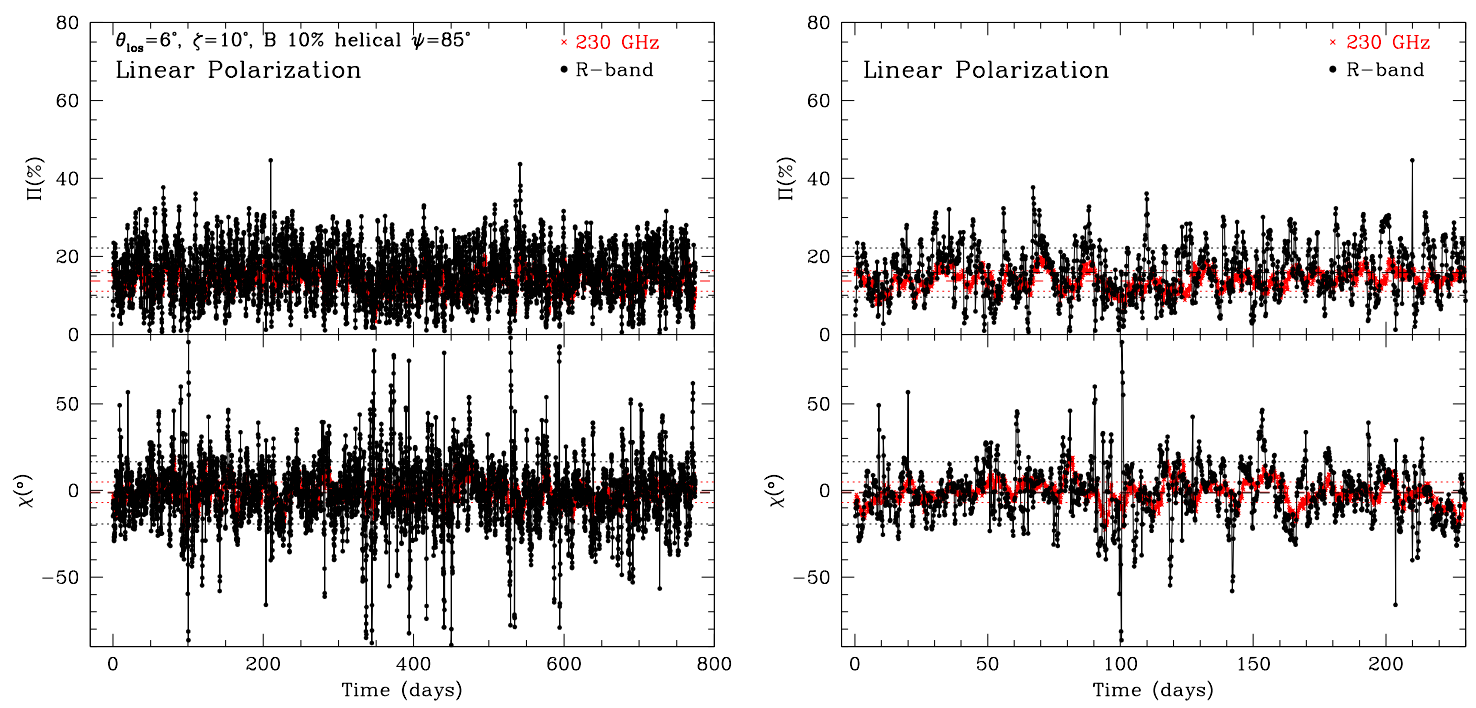

Figure 5: Simulated optical linear polarization vs. time produced by the TEMZ model for parameters similar to those of BL Lacertae. Left: Full run of 5000 time steps; right: expansion of the section containing the first flare seen in Fig. 4. Compare with Fig. 3, bottom two panels.

One way of discerning between the TEMZ and other models is the prediction of wavelength- 
dependent optical polarization. Figure 6 plots a histogram of the spectral index between R and B band of the degree $\Pi$ of polarization as a function of $\Pi$ from the TEMZ run that generated Figures 4 and 5. Although there is a small favoritism for the B-band polarization to be stronger, a substantial minority of measurements should find the R-band polarization to be higher as well. This provides a definitive test of scenarios that rely on stochastic magnetic field directions to produce short timescale fluctuations of the synchrotron radiation. In addition, an increase in the number of the highestenergy electrons can occur when a cell with a particularly high magnetic field and density, and with favorable orientation of the field, crosses the shock. This causes a rapid X-ray synchrotron and VHE $\gamma$-ray flare, as well as a flattening of the polarized flux density spectrum, since much of the emission at that time should come from a single cell or a few cells. Data by the author's group and from the Steward Observatory polarization spectral monitoring [18] indeed provide evidence for the predicted wavelength dependence [11]. The author's group plans to expand its monitoring of the multi-color optical polarization of blazars to confirm and study this trend.

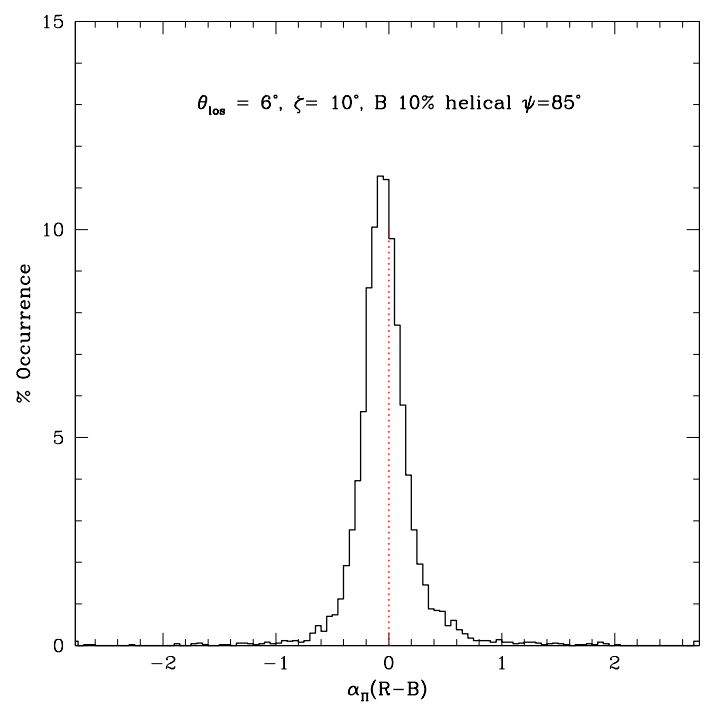

Figure 6: Wavelength dependence (between B- and R-band) of the optical polarization of the same TEMZ model run whose time behavior is displayed in Figs. 4 and 5. A negative value of the spectral index $\alpha_{\Pi}$ of the degree of polarization corresponds to a flatter polarized flux density spectrum than the total flux density spectrum. Note that flatter polarized spectra are more common.

\section{Conclusions}

Blazar data contain indications of physical processes that generate both order and disorder in the light curves and polarization behavior. The hypothesis that this is caused by the superposition of turbulence on systematic structures such as nearly cylindrical jets and shock waves, appears promising: current simulations bear resemblance to observed timing data. Of course, much work remains to be done. For example, more physical processes such as moving shocks and particle acceleration from major magnetic reconnection events, could improve the realism of models such 

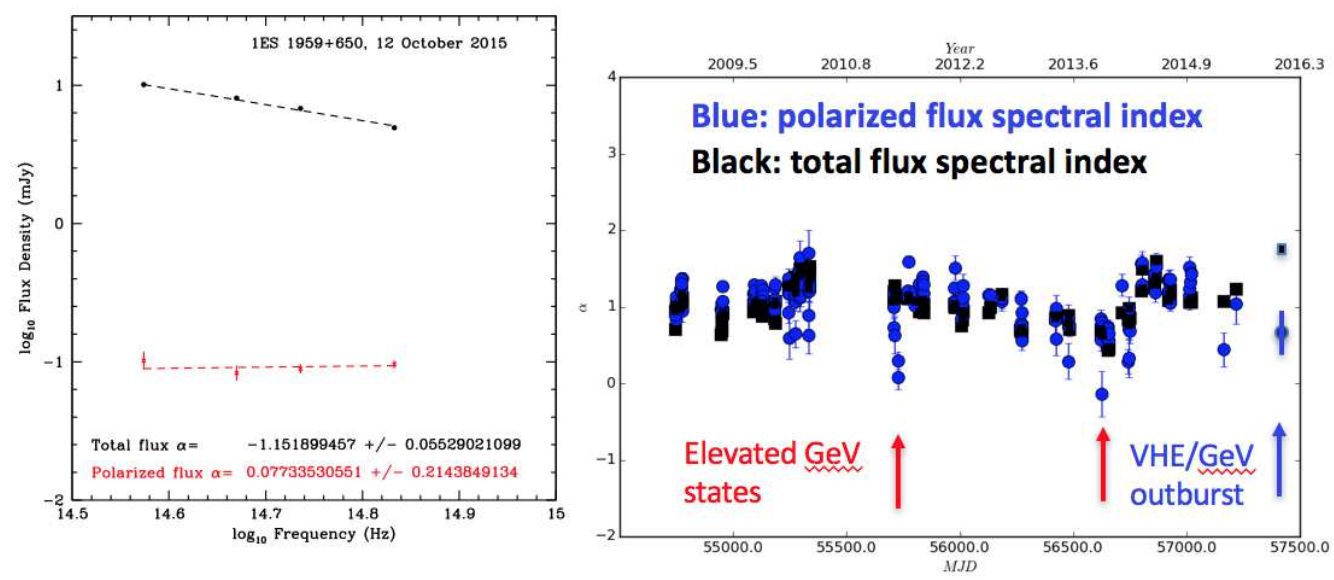

Figure 7: Polarized and total flux density spectral indices for the VHE emitting BL Lac object 1ES 1959+650. The polarized flux spectrum flattens relative to the total flux spectrum during prominent $\gamma$-ray outbursts. From data presented in [11]

as TEMZ. Other researchers are combining hydrodynamical and magneto-hydrodynamical simulations of turbulence in jets with emission calculations ([17, 20]). As these models become more sophisticated, statistical comparison of the variations and correlations can be compared with similar analyses of the data in order to determine whether they can explain the full range of blazar behavior.

\section{References}

[1] F. Acero et al., Fermi Large Area Telescope Third Source Catalog, Astrophys. J. Suppl. 218 (2015) 23

[2] D. Blinov et al., RoboPol: do optical polarization rotations occur in all blazars?, MNRAS 462 (2016) 1775

[3] V. Calafut, V. \& P.J. Wiita, Modeling the Emission from Turbulent Relativistic Jets in Active Galactic Nuclei, J. Astrophys. \& Astron. 36 (2014) 255

[4] T.V. Cawthorne, Polarization of synchrotron radiation from conical shock waves, MNRAS 367 (2006) 851

[5] T.V. Cawthorne, S.G. Jorstad, \& A.P. Marscher, Polarization Structure in the Core of 1803+784: A Signature of Recollimation Shocks?, Astrophys. J. 772 (2013) 14

[6] R. Chatterjee et al., Similarity of the Optical-Infrared and Gamma-Ray Time Variability of Fermi Blazars, Astrophys. J. $\mathbf{7 4 9}$ (2012) 191

[7] T.W. Jones, Polarization as a Probe of Magnetic Field and Plasma Properties of Compact Radio Sources - Simulation of Relativistic Jets, Astrophys. J. 332 (1988) 678

[8] S.G. Jorstad et al., Polarimetric Observations of 15 Active Galactic Nuclei at High Frequencies: Jet Kinematics from Bimonthly Monitoring with the Very Long Baseline Array, Astron. J. 130 (2005) 1418

[9] S.G. Jorstad et al., Multiwaveband Polarimetric Observations of 15 Active Galactic Nuclei at High Frequencies: Correlated Polarization Behavior, Astron. J. 134 (2007) 799 
[10] S.G. Jorstad et al., A Tight Connection between Gamma-Ray Outbursts and Parsec-Scale Jet Activity in the Quasar 3C 454.3, Astrophys. J. 773 (2013) 147

[11] M.P. Malmrose, The Near-Infrared to Ultraviolet Spectral Energy Distributions of Gamma-Ray Bright Blazars, Ph.D. Dissertation, Boston University (2016)

[12] A.P. Marscher, Turbulent, Extreme Multi-Zone Model for Simulating Flux and Polarization Variability in Blazars, Astrophys. J. 780 (2014) 87

[13] A.P. Marscher et al., The Inner Jet of an Active Galactic Nucleus as Revealed by a Radio to Gamma-ray Outburst, Nature 452 (2008) 966

[14] A.P. Marscher et al., Probing the Inner Jet of the Quasar PKS 1510-089 with Multi-waveband Monitoring during Strong Gamma-ray Activity, Astrophys. J. Lett. 710 (2010) L126

[15] A.P. Marscher \& W.K. Gear, Models for High-Frequency Radio Outbursts in Extragalactic Sources with Application to the Early 1983 Millimeter to Infrared Flare of 3C 273, Astrophys. J. 298 (1985) 114

[16] R. Narayan \& T. Piran, Variability in blazars: clues from PKS 2155-304, MNRAS 420 (2012) 604

[17] M. Pollack, D. Pauls, \& P.J. Wiita, Variability in Active Galactic Nuclei from Propagating Turbulent Relativistic Jets, Astrophys. J. 820 (2016) 12

[18] P.S. Smith, Sharp Polarimetric Eyes: More Trees than Forest?, Galaxies 4 (2016) 27

[19] E.J. Summerlin \& M.G. Baring, Diffusive Acceleration of Particles at Oblique, Relativistic, Magnetohydrodynamic Shocks, Astrophys. J. 745 (2012) 63

[20] J.R. Webb, Multi-Frequency Blazar Micro-variability as a Tool to Investigate Relativistic Jets, Galaxies 4 (2016) 15

[21] A.E. Wehrle et al. Erratic Flaring of BL Lac in 2012-2013: Multiwavelength Observations, Astrophys. J. 816 (2016) 53

[22] K.E. Williamson et al. Comprehensive Monitoring of Gamma-ray Bright Blazars. I. Statistical Study of Optical, X-ray, and Gamma-ray Spectral Slopes, Astrophys. J. 789 (2014) 135 